\title{
Cross-Spectrum Resource Management and Access for Heterogeneous Cellular Networks
}

\author{
SHEN Jia ${ }^{\# 1}$, LÜ Xiangyou ${ }^{* 2}$, LI Huafei ${ }^{* 3}, \mathrm{XU} \mathrm{Fei}^{\# 4}$ \\ ${ }^{\#}$ Radio \& Mobile Department, Research Institute of Telecommunications Transmission, MIIT of China \\ NO.11, Yue Tan Nan Jie, Xicheng District, Beijing, 100045, CHINA \\ ${ }^{1}$ shenjia@mail.ritt.com.cn \\ ${ }^{4}$ xufei@mail.ritt.com.cn \\ "School of information and Communication Engineering, Beijing University of Post and Telecommunications \\ Xi Tu Cheng Road, Haidian District, Beijing, 100876, CHINA \\ ${ }^{2} 02$ jordan@bupt.cn \\ ${ }^{3}$ Ihf4525@bupt.cn
}

\begin{abstract}
This paper introduces a resource management scheme for a heterogeneous cellular network. In a heterogeneous network using multiple frequency bands, the scheme can schedule the users into the band according to the user's channel conditions, e.g. geometry and mobility. According to the simulation results, the new scheme can provide a substantial gain over the independent resource allocation in each band.
\end{abstract}

Keywords - spectrum sharing, heterogeneous network, resource management.

\section{INTRODUCTION}

The emergence of broadband wireless communications requires more frequency resource. However, the increasing requirements can hardly be met due to the shortage of usable spectrum. This fact encourages a more flexible usage of spectrum via spectrum sharing and cognitive radio technologies.

Two kinds of spectrum sharing have being discussed: Sharing between a cellular system and another system [1-3] (e.g. a broadcasting system, unlicensed system) and sharing between multiple spectrums used by a cellular operator. This paper focuses on the second scenario. In a traditional scheme, a cellular operator use different spectrums to deploy different systems. The systems in different spectrums would work independently. However, when a single frequency band cannot meet the spectrum requirement of a broadband cellular system, the schemes of operating a system across multiple non-continuous bands are considered.

Although the multiple bands are owned by the same operator, the different bands have different features, and then different values. Some bands with good channel propagation features and smaller interferences are very precious resource. Some bands having relatively bad channel features are less valuable, although they often have more sufficient amount. Hence, a more effective and reasonable sharing of good spectrums are necessary. Some economic approaches (e.g. Game Theory) are introduced for the sharing between spectrums with different qualities [4-9].

In this paper, we propose a cross-spectrum resource management scheme with a principle similar to "Pricing" schemes. Using this scheme, a BS (Base Station) "auctions" the lower frequency spectrum to users with higher prices, while "sells" higher frequency spectrum to users with lower prices ("The higher the quality, the higher the price."). This approach actually attracts those users who can use either the higher or the lower band to "buy" the high band with a cheaper price. Thus the precious low frequency spectrum can be saved for the users who can only be accommodated in the low frequency bands. Compared to the traditional scheme in which the high and low frequency bands are allocated separately (auctioned with equal prices), the proposed scheme can obtain capacity gain because of the better usage of low frequency bands.

The rest of the paper is organized as following: Section II describes the multi-band heterogeneous cellular network architecture we consider in the paper. In Section III, the crossspectrum resource management scheme is introduced, including how to schedule a user to a suitable band, and the relative common channel structures. In Section IV, the capacity performance comparison between the proposed scheme and the traditional scheme is demonstrated with simulation results. Section V finally concludes the paper.

\section{Heterogeneous CEllular Networks}

Future broadband mobile systems require an increasingly larger amount of spectrum. For example, the IMT-Advanced systems need at least $40-60 \mathrm{MHz}$ spectrum to meet the $1 \mathrm{Gbps}$ data rate. However, the spectrum cannot easily been found within a single band, especially for the low frequency bands. Therefore, the non-continuous spectrum aggregation is considered that can aggregate multiple frequency bands to obtain a wider operating band [10]. A typical scenario is that an operator has some lower-frequency bands (e.g. $<1 \mathrm{GHz})$ as well as some higher-frequency bands (e.g. $>2 \mathrm{GHz}$ ). The channel characteristics of a lower frequency band and that of a higher frequency band are quite different. A lower band in general provides a much better coverage and mobility performance than a higher band. However, the lower frequency resource is always more precious than the higher one, i.e. the amount of the lower frequency resource is quite limited. 
In this paper, we consider a heterogeneous cellular network. Fig. 1 illustrates an example of the dual-band (considering the simplest scenario) radio access network (RAN). The operations in the two bands should be supported with the same set of BS sites.
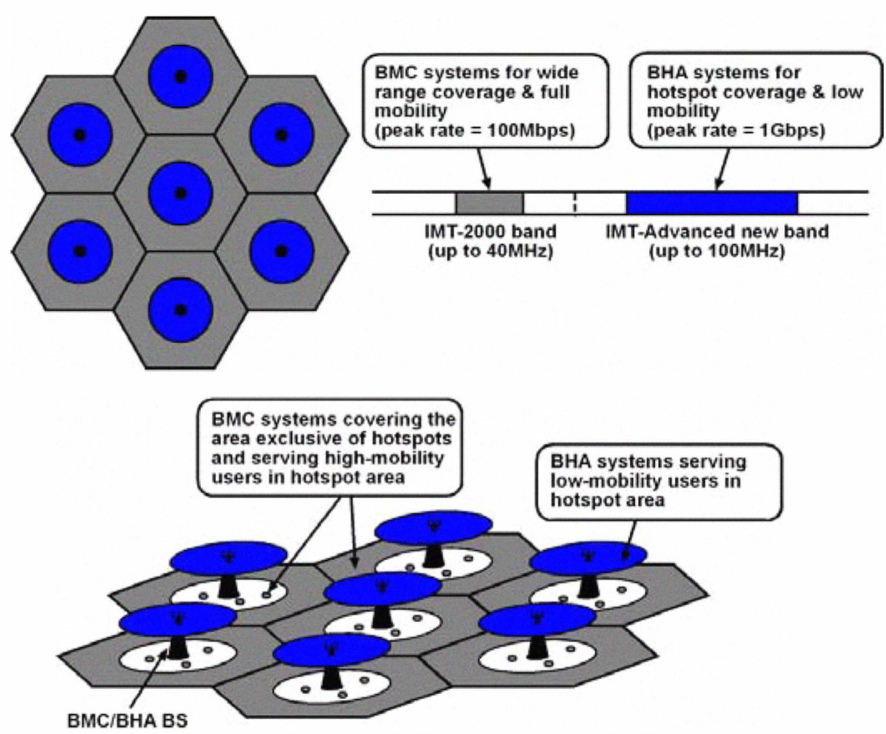

Fig. 1 Dual-band heterogeneous cellular network architecture

The lower band (LB) and upper band (UB) have significantly different characteristics. The upper band operation (UBO) can provide ultra large system bandwidth (up to $100 \mathrm{MHz}$ ) needed for high peak data rate and system capacity, nevertheless cannot provide wide area coverage or support high mobility. The lower band operation (LBO) can provide wide area coverage and support high mobility, nevertheless can only provide a relatively small bandwidth (e.g. $5-40 \mathrm{MHz}$ ) to achieve a moderate peak data rate and system capacity.

Based on the dual-band spectrum baseline, the proposed resource management scheme should be adopted to enable the efficient operations in the two bands. It should be noted that IMT-Advanced system requirements could be fulfilled with the joint operations in the two bands, although the operation in each band may only fulfil a part of the requirements. The key features of the operations in the two bands are summarized respectively as following:

Lower band operation (LBO):

- System bandwidth: 5 40MHz for unicast/multicast system;

- Provide wide area coverage;

- Support high mobility (up to $500 \mathrm{~km} / \mathrm{h}$ ).

Upper band operation (UBO):

- System bandwidth: 20 100MHz for unicast system;

- Provide local area coverage;

- Support low mobility.

\section{CROSS-SPECTRUM RESOURCE MANAGEMENT}

The UBO provides a smaller coverage range than the $\mathrm{LBO}$, hence only covers the center area of the cells (as shown in Fig. 1). The UB is used to provide ultra high data rate (up to $1 \mathrm{Gbps}$ ) and high system capacity in these "hotspots", and only serve the low-mobility users. The LB is used to provide full coverage and to serve high-mobility users.

The operations in the two bands are not realized in an independent manner, but performed jointly. The users are scheduled cross the two bands based on a "cross-spectrum scheduling" operation, following the criteria below:

- If a user is located in the "hotspot" area, and with a low mobility, it is served with the UB.

- If a user is located outside the hotspot area, it is served with the LB.

- If a user is located in the hotspot area, but with a high mobility, it is served with the LB.

The upper band covers the hotspots ("cell-center" area) for fixed/low-mobility users. Hence the LB can concentrate its "precious" frequency resource on "cell-edge" area and highmobility users, and a higher system throughput can be achieved in cell edge area. The load balancing between the two bands are supported for efficient use of spectrum.

In a dense urban area, the legacy BSs are deployed in a siteto-site distance of $\sim 100 \mathrm{~m}$. In this case, the upper band may also provide a full coverage for all low-mobility users, while the LB is mainly used to serve high-mobility users. In this case, the UBO is also performed in a cellular manner, e.g. supporting inter-cell handover and inter-cell interference mitigation.

In a normal urban, suburban or rural area, the site-to-site distance could be too large for the the UBO to provide a full coverage. In this case, the UBO should be set where a heavy broadband access load arises. If the legacy sites are not located in a proper position to cover the heavy-loaded area, new sites (BS or relaying stations) may be needed (it will be relatively easy to set new sites in suburban and rural areas).

In a rural area without ultra high data rate/system capacity requirements, the UBO could absent. All paragraphs must be indented. All paragraphs must be justified, i.e. both leftjustified and right-justified.

Cell search is the procedure by which a user terminal acquires time and frequency synchronization with a cell and detects the specific information of that cell. Synchronization operation is mainly performed based on $\mathrm{SCH}$ (synchronization channel). Cell identification operation could be performed based on $\mathrm{SCH}, \mathrm{BCH}$ (broadcast channel) or/and pilots.

The primary purpose of the $\mathrm{SCH}$ is to enable acquisition of the frequency and received timing, i.e., at least the $\mathrm{SCH}$ symbol timing and frequency of the downlink signal.The Downlink SCH occupies the central part of these transmission bandwidths, and the $\mathrm{SCH}$ structure is based on the constant bandwidth regardless of the overall transmission bandwidth of the cell.

Aside from the SCH symbol timing and frequency information, the $\mathrm{BCH}, \mathrm{SCH}$ or some additional channels, such as the pilots, can be distributed to carry the cell search sequences in the way of FDM or CDM, to help the user terminal acquire at least the following cell-specific information:

- Cell ID 
- Radio frame timing information when this is not directly given by the $\mathrm{SCH}$ timing

- Information regarding the antenna configuration of the cell (number of transmitter antennas),

To obtain reliable detection in multipath scenarios, differential structures and correlation estimators could be employed in cell search, for the differential structure is known to be robust in multipath fading channels.

For a system with both LBO and UBO modes, the cell search and $\mathrm{SCH} / \mathrm{BCH}$ structure can be jointly optimized to more effectively support fast cell search and inter-mode handover/re-selection. Two possible optimizations under consideration are:

- Staggered allocation of $\mathrm{SCH} / \mathrm{BCH}$ in time domain between LBO and UBO modes will facilitate a faster $\mathrm{SCH} / \mathrm{BCH}$ joint cell search because a UT can finish a cell search to LBO as well as UBO cells within one $\mathrm{SCH} / \mathrm{BCH}$ period. This results in an easier inter-mode measurement and handover/re-selection because a UT can perform tuning-off measurement of $\mathrm{SCH} / \mathrm{BCH}$ of the other mode without missing that of current mode.

- Shared SCH between LBO and UBO modes (i.e. only allocate $\mathrm{SCH}$ in one of the two modes).

Two $\mathrm{SCH} / \mathrm{BCH}$ structures and their relative cell search procedures are given below:

\section{Structure 1:}

As shown in Fig. 3, LBO and UBO cells both transmit SCH as well as $\mathrm{BCH}$. The merit of structure 1 the possibility of fast acquisition of cell ID for either the UBO or LBO mode. However, if a UT is not located in the coverage of UBO mode or in a high mobility, the SCH of the UBO mode could not be successfully detected. Since a UT does not have the a priori knowledge of its location, the UT is not able to select the proper SCH from the two. Hence a UT should first perform synchronization via the LBO $\mathrm{SCH}$. The UBO $\mathrm{SCH}$ can be used for a UT in the UBO mode to track the UBO timing and frequency.

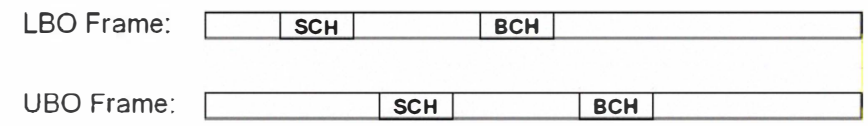

Fig. 3 Structure 1 for $\mathrm{SCH} / \mathrm{BCH}$ allocation in $\mathrm{LBO} / \mathrm{UBO}$ dual-mode system

A cell search procedure for the $\mathrm{SCH} / \mathrm{BCH}$ structure 1 shown in Fig. 4 (shown in Fig. 3) can be described as below:

- A UT first detects the SCHs in the LB to achieve the downlink timing and frequency synchronization to both the LBO and UBO mode, identifies the cell IDs of the neighbouring LBO cells, and obtains basic information about these cells (at least including $\mathrm{CP}$ length, MIMO configurations, and $\mathrm{BCH}$ transmission parameters).

- The UT detects the SCHs in the UB to identify the cell IDs of the neighbouring UBO cells, track the timing/frequency of the UBO mode, and obtain basic information about these cells (at least including CP length, MIMO configurations, and $\mathrm{BCH}$ transmission parameters).

The UT detects the BCHs of the neighbouring LBO cells to obtain additional information about these cells.

- The UT detects the BCHs of the neighbouring UBO cells to obtain additional information about these cells.

- The UT makes cell selection decisions based on the search results, e.g. access the LBO or the UBO mode, which cell to access.

- The UT raises an uplink access attempt to the selected cell.

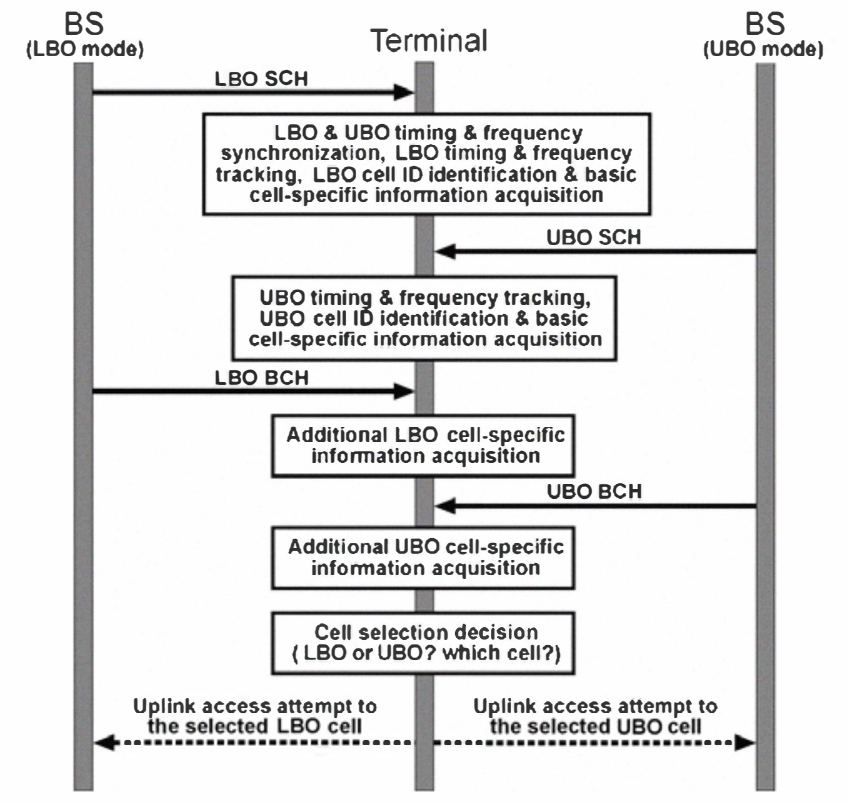

Fig. 4 Cell search procedure for $\mathrm{SCH} / \mathrm{BCH}$ structure 1 (shown in Fig. 4)

\section{Structure 2:}

As shown in Fig. 5, LBO and UBO cells both transmit $\mathrm{BCH}$, while only $\mathrm{LBO}$ cells transmit $\mathrm{SCH}$. $\mathrm{BCH}$ of $\mathrm{LBO}$ and UBO are staggered placed in time domain. This structure is at least applicable for the scenario where a LBO cell and a UBO cell are co-located in a same BS.

LBO Frame:

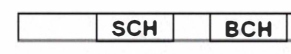

UBO Frame: $\mathrm{BCH}$

Fig. 5 Structure 2 for $\mathrm{SCH} / \mathrm{BCH}$ allocation in LBO/UBO dual-mode system

In case that only the $\mathrm{LBO}$ mode carries $\mathrm{SCH}$, a UT always searches the LBO cells to obtain and resume the connection to the system. Then the UT could be re-scheduled to the UBO mode if it is located in the UBO coverage area and in a low mobility. If relative timing synchronization is maintained between the UBO and the LBO modes, a UT can synchronize with one of the two modes, and then naturally achieves synchronization with the other.

This structure avoids the SCH overhead and complexity of timing synchronization for the UBO mode. However, this 
implies that the no SCH can be used in the UBO mode for frequency synchronization and tracing. This may bring difficulties to UBO frequency synchronization unless one oscillator is used to generate timing for LBO and UBO in the same BS. A possible solution is that a UT in the UBO mode can perform the periodical carrier frequency tracing using the pilots of UBO mode.

A cell search procedure for the $\mathrm{SCH} / \mathrm{BCH}$ structure 2 (shown in Fig. 5) shown in Fig. 6 can be described as below:

- A UT first detects the SCHs in the LB to achieve the downlink timing and frequency synchronization to both the LBO and UBO sub-system, identifies the cell IDs of the neighboring LBO cells, and obtain basic information about these cells (at least including $\mathrm{CP}$ length, MIMO configurations, and $\mathrm{BCH}$ transmission parameters). $\mathrm{LBO}$ $\mathrm{SCH}$ could also transmit basic information about neighboring UBO cells (e.g. their $\mathrm{BCH}$ configurations or pilot structures) to assist the $\mathrm{BCH} /$ pilot-based cell search to the UBO mode.

- The UT detects the BCHs of the neighboring LBO cells to obtain additional information about these cells. LBO $\mathrm{BCH}$ could also transmit basic information about neighboring UBO cells (e.g. their $\mathrm{BCH}$ configurations or pilot structures) to assist the $\mathrm{BCH} /$ pilot-based cell search to the UBO mode.

- The UT detects the BCHs or pilot channels of the neighboring UBO cells to identify their cell IDs, track the timing/frequency of the UBO sub-system in Active state, and obtain additional information about these cells.

- The UT makes cell selection decisions based on the search results, e.g. access the LBO or the UBO subsystem, which cell to access.

- The UT raises an uplink access attempt to the selected cell.

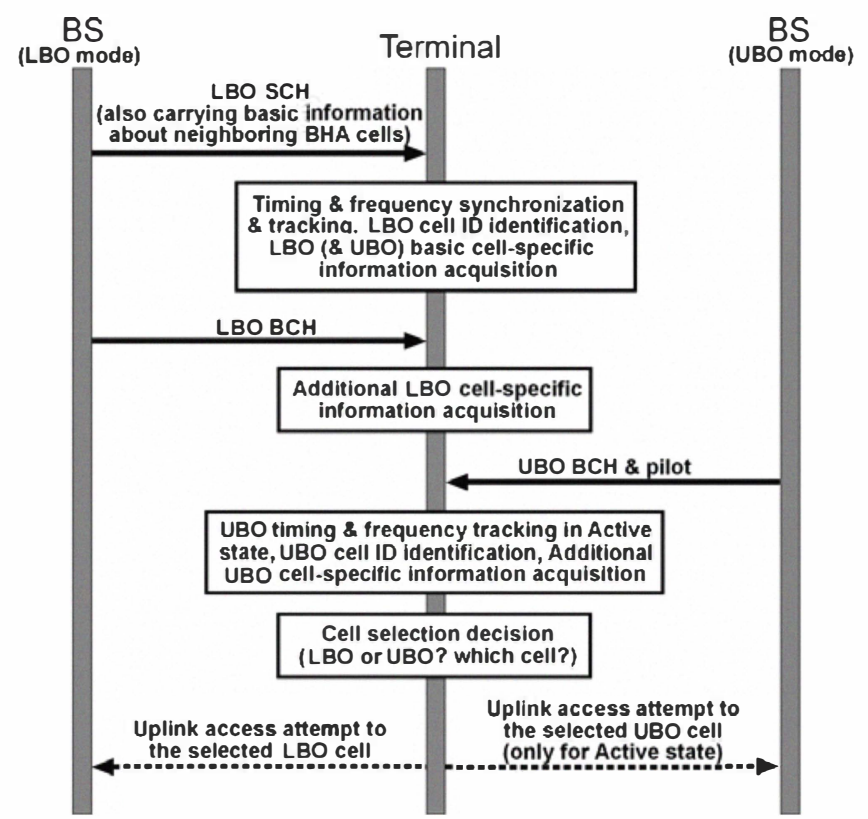

Fig. 6 Cell search procedure for $\mathrm{SCH} / \mathrm{BCH}$ structure 2 (shown in Fig. 5)

\section{SIMULATION RESULTS AND ANALYSIS}

In this section, we simulate the performance of the proposed scheme. We consider a 3GPP LTE (Long-Term Revolution) system with the 19-BS 57-cell layout (each BS contains 3 cells (sectors)), and the downlink spectrum efficiency (bit/s/Hz/cell) of the center cell is simulated. In each cell, the UBO BS and the LBO BS co-located (just as shown in Fig. 1). Since the LBO has a better coverage than UBO. The SINR of user at the edge of the LBO cell will be much larger than the UBO cell. Each cell has $10 \mathrm{MHz}$ spectrum in UB and $10 \mathrm{MHz}$ in LB. The BSs employ closedloop precoding spatial multiplexing (Transmission Mode 4 in [11-12]) in downlink transmission. The carrier frequency of LBO is set to $800 \mathrm{MHz}$, and that of UBO is set to $2 \mathrm{GHz}$. We drop 20 users in a cell (the cell uses UBO and LBs). It is assumed that the LB and the UB can serve up to 10 users respectively. Each band will try to serve as many users as possible. However, if over 10 users try to access to any band of the two, the band will select the 10 users with highest SINRs, while reject the other users.

The simulation parameters are summarized in Table I [13].

TABLE I

SIMULATION ASSUMPTIONS

\begin{tabular}{|c|c|c|}
\hline \multicolumn{2}{|c|}{ Parameter } & Value \\
\hline \multicolumn{2}{|c|}{ Number of Cells } & 19 \\
\hline \multicolumn{2}{|c|}{ Number of Sectors per Cell } & 3 \\
\hline \multicolumn{2}{|c|}{ Inter-site distance $(\mathrm{m})$} & 1732 \\
\hline \multicolumn{2}{|c|}{ Antenna Configuration } & $4 \times 2$ \\
\hline \multicolumn{2}{|c|}{ Antenna type } & Independence \\
\hline \multicolumn{2}{|c|}{ Centre Frequency (LBO) } & $800 \mathrm{MHz}$ \\
\hline \multicolumn{2}{|c|}{ Centre Frequency (HBO) } & $2 \mathrm{GHz}$ \\
\hline \multicolumn{2}{|c|}{ Traffic model } & Full Buffer \\
\hline \multicolumn{2}{|c|}{ BS transmit power } & $46 \mathrm{dBm}$ \\
\hline \multicolumn{2}{|c|}{ Lognormal Shadowing } & Standard deviation: $10 \mathrm{~dB}$ \\
\hline \multirow[t]{2}{*}{$\begin{array}{l}\text { Shadowing } \\
\text { Correlation }\end{array}$} & \begin{tabular}{|l|} 
Between \\
cells
\end{tabular} & 0.5 \\
\hline & \begin{tabular}{|l|} 
Between \\
sectors \\
\end{tabular} & 1 \\
\hline \multicolumn{2}{|c|}{ UE Noise Figure } & $9 \mathrm{~dB}$ \\
\hline \multicolumn{2}{|c|}{ MS Antenna Gain } & $0 \mathrm{dBi}$ \\
\hline \multicolumn{2}{|c|}{ BS Antenna Gain } & $14 \mathrm{dBi}$ \\
\hline \multicolumn{2}{|c|}{ Path-Loss } & $128.1+37.6 \log 10(\mathrm{R}), \mathrm{R}$ in $\mathrm{km}$ \\
\hline \multicolumn{2}{|c|}{ Thermal Noise Density } & $-174 \mathrm{dBm} / \mathrm{Hz}$ \\
\hline \multicolumn{2}{|c|}{$\begin{array}{l}\text { Antenna Bore-sight points } \\
\text { toward flat side of cell (for } \\
\text { 3-sector sites with fixed } \\
\text { antenna patterns) }\end{array}$} & \\
\hline \multicolumn{2}{|c|}{$\begin{array}{l}\text { BS Antenna } \\
\text { pattern(horizontal) } \\
\text { (For 3-sector cell sites with } \\
\text { fixed antenna patterns) }\end{array}$} & $\begin{array}{l}A(\theta)=-\min \left[12\left(\frac{\theta}{\theta_{3 d B}}\right)^{2}, A_{m}\right] \\
\theta_{3 d B}=70 \text { degrees, } A_{m}= \\
20 \mathrm{~dB}\end{array}$ \\
\hline \multicolumn{2}{|c|}{$\begin{array}{l}\text { Minimum distance between } \\
\text { UE and Cell }\end{array}$} & $>=35$ meters \\
\hline \multicolumn{2}{|c|}{$\begin{array}{l}\text { Users dropped uniformly in } \\
\text { entire cell }\end{array}$} & \\
\hline \multicolumn{2}{|c|}{ UE number per sector } & $10 \sim 20$ \\
\hline
\end{tabular}


Two user scheduling schemes are compared:

- Scheme 1 (tradition scheme): Each UT first measures SINR of LBO and that of UBO respectively. Then the UT always selects and tries to access the band with the higher SINR.

Band $= \begin{cases}U B O, & \text { if } S I N R_{U B O} \geq S I N R_{L B O} \\ L B O, & \text { if } S I N R_{U B O}<S I N R_{L B O}\end{cases}$

- Scheme 2 (proposed scheme): Each UT first measures SINR of LBO and that of UBO respectively. Then the UT will add a "Price" weight $W_{\text {Price }}$ to $\operatorname{SINR}_{U B O}$ :

$$
\operatorname{SINR}_{U B O}{ }^{\prime}=S I N R_{U B O}+W_{\text {Price }}
$$

Then the UT selects and tries to access to the better band with the higher SINR, while using $S_{I N R_{U B O}}$ as the UBO SINR. In the simulation, we assume is $W_{\text {Price }}=1.5 \mathrm{~dB}$. This is an experienced value. It is found this is the most suitable "Price" to balance the load in UB and LB.

$$
\text { Band }= \begin{cases}U B O, & \text { if } S_{I N R_{U B O}}{ }^{\prime} \geq S I N R_{L B O} \\ L B O, & \text { if } S_{I N R_{U B O}}{ }^{\prime}<S I N R_{L B O}\end{cases}
$$

The system-level simulation results are shown in Table II. The results show that the proposed scheduling scheme can provide $8.7 \%$ gain over the traditional one in average cell spectrum efficiency.

TABLE II

THE CELL SPECTRUM EFFICIENCY OF TWO SCHEDULING SCHEMES

\begin{tabular}{|c|c|c|}
\hline & $\begin{array}{c}\text { Scheme 1 } \\
\text { (Traditional) }\end{array}$ & $\begin{array}{c}\text { Scheme 2 } \\
\text { (Proposed) }\end{array}$ \\
\hline $\begin{array}{c}\text { Average cell spectrum } \\
\text { efficiency } \\
\text { (bit/s/Hz/cell) }\end{array}$ & 1.49 & 1.62 \\
\hline $\begin{array}{c}\text { Gain of Scheme 2 over } \\
\text { Scheme 1 }\end{array}$ & $0 \%$ & $8.7 \%$ \\
\hline
\end{tabular}

We can analyze the above results as following: Scheme 1 cannot achieve the balance load in UB and LB. In general, over 10 users try to access the LB and some users are rejected, while the UB is only partly used because only less than 10 users select the UB. Scheme 2 uses the "Price" weight to increase the selecting threshold for LBO. This equivalently moves some users from LB to UB, and finally achieves the load balancing between the two bands.

\section{CONCLUSIONS}

This paper proposed a joint resource management scheme for a multi-band heterogeneous network. By set a higher "Price" for the lower frequency band when "auctioning" the spectrum to users, the values of the higher and lower bands are balanced. The proposed scheme can provide a substantial capacity gain over the traditional one. Furthermore the relative joint scheduling protocols, $\mathrm{SCH} / \mathrm{BCH}$ structures and joint cell search procedures are also proposed.

\section{ACKNOWLEDGMENT}

The research work introduced in this paper was supported by a grant from the National High Technology Research and Development Program of China (863 Program) (No. 2008AA01Z213): "Study on Heterogeneous RAN architecture and protocol based on multi-layer overlay-structuring technology".

\section{REFERENCES}

[1] Bruce Fette, Cognitive Radio Technology: Newnes, Aug. 2006

[2] J. Mitola, Cognitive Radio for Flexible Mobile Multimedia Communications, Proc. of IEEEWorkshop on Mobile Multimedia Communication, pp. 3-10, 1999

[3] S. Haykins, Cognitive Radio: Brain-Empowered Wireless Communications, IEEE Journal on Selected Areas in Communications, Vol. 23, No. 2, pp. 201-220, Feb. 2008.

[4] J. Zhang and Q. Zhang, Stackelberg Game for Utility-Based Cooperative Cognitive Radio Networks, MobiHoc' 09, May 2009.

[5] V. Rodriguez, K. Moessner and R. Tafazolli, Auction Driven Dynamic Spectrum Allocation:

[6] Optimal Bidding, Pricing and Service Priorities for Multi-rate, Multiclass CDMA, IEEE PIMRC 2005, vol. 3, pp. 1850-1854, Sept. 2005.

[7] Y. Xing, R. Chandramouli and C. M. Cordeiro, Price Dynamics in Competitive Agile Spectrum Access Markets, IEEE Journal on Selected Areas in Communication, vol. 25, no. 3, pp. 613-621, Apr. 2007

[8] J. Huang, R. A. Berry and M. L. Honig, Auction-Based Spectrum Sharing, ACM Mobile Networks and Applications J., vol. 11, no. 3, pp. 405-418, June 2006.

[9] S. Gandhi, C. Buragohain L. Cao, H. Zheng and S. Suri, A General Framework for Wireless Spectrum Auctions, IEEE International Symp. New Frontiers in Dynamic Spectrum Access Networks (DySPAN '07),

[10] 3GPP TR 36.814 Evolved Universal Terrestrial Radio Access (EUTRA); Further advancements for E-UTRA Physical layer aspects

[11] 3GPP TS 36.213 Evolved Universal Terrestrial Radio Access (EUTRA); Physical Layer Procedure (Release 8)

[12] 3GPP TS 36.211 Evolved Universal Terrestrial Radio Access (EUTRA); Physical Channels and Modulation (Release 8)

[13] ITU M.2135. Guidelines for evaluation of radio interface technologies for IMT-Advanced. 